Meta

Journal des traducteurs

Translators' Journal

\title{
Le traducteur et ses cibles : lectures analytique et empathique en adaptation publicitaire
}

\section{Hugo Vandal-Sirois}

Volume 60, numéro 1, avril 2015

URI : https://id.erudit.org/iderudit/1032397ar

DOI : https://doi.org/10.7202/1032397ar

Aller au sommaire du numéro

Éditeur(s)

Les Presses de l’Université de Montréal

ISSN

0026-0452 (imprimé)

1492-1421 (numérique)

Découvrir la revue

Citer cet article

Vandal-Sirois, H. (2015). Le traducteur et ses cibles : lectures analytique et empathique en adaptation publicitaire. Meta, 60(1), 3-17.

https://doi.org/10.7202/1032397ar
Résumé de l'article

Pour toucher un public et parvenir à modifier son comportement, le traducteur publicitaire doit démontrer une excellente connaissance de la rédaction publicitaire, d'autant plus que le public cible et le contexte communicationnel de la traduction peuvent différer de façon significative de ceux de la publicité source. En tant qu'agent culturel de l'annonceur, le traducteur publicitaire repère les éléments textuels et paratextuels qui nuiront à l'efficacité du message et apporte les ajustements nécessaires. Dans ce but, il effectuera une double lecture de la publicité originale pour saisir l'ensemble des enjeux communicationnels et orienter sa démarche traductive. D’abord, la lecture analytique correspond à un premier travail de décodage rationnel, influencé par les connaissances et les aptitudes du traducteur en rédaction publicitaire, ainsi que par son environnement de travail, les ressources à sa portée et le contexte communicationnel. Ensuite, la lecture empathique permet de comprendre comment fonctionne le message à traduire du point de vue émotionnel pour ensuite recréer l'effet voulu, en considérant l'identité du public cible ainsi que les aspects culturels et l'objectif de la communication. Cet article montre que ces deux lectures, respectivement raisonnée et émotive, s'avèrent essentielles pour l'opération à la fois rationnelle et créative qu'est l'adaptation publicitaire.
Ce document est protégé par la loi sur le droit d'auteur. L’utilisation des services d'Érudit (y compris la reproduction) est assujettie à sa politique d'utilisation que vous pouvez consulter en ligne.

https://apropos.erudit.org/fr/usagers/politique-dutilisation/ 


\title{
Le traducteur et ses cibles: lectures analytique et empathique en adaptation publicitaire
}

\author{
HUGO VANDAL-SIROIS \\ Université de Montréal, Montréal, Canada \\ hugo.vandal-sirois@umontreal.ca
}

\section{RÉSUMÉ}

Pour toucher un public et parvenir à modifier son comportement, le traducteur publicitaire doit démontrer une excellente connaissance de la rédaction publicitaire, d'autant plus que le public cible et le contexte communicationnel de la traduction peuvent différer de façon significative de ceux de la publicité source. En tant qu'agent culturel de l'annonceur, le traducteur publicitaire repère les éléments textuels et paratextuels qui nuiront à l'efficacité du message et apporte les ajustements nécessaires. Dans ce but, il effectuera une double lecture de la publicité originale pour saisir l'ensemble des enjeux communicationnels et orienter sa démarche traductive. D'abord, la lecture analytique correspond à un premier travail de décodage rationnel, influencé par les connaissances et les aptitudes du traducteur en rédaction publicitaire, ainsi que par son environnement de travail, les ressources à sa portée et le contexte communicationnel. Ensuite, la lecture empathique permet de comprendre comment fonctionne le message à traduire du point de vue émotionnel pour ensuite recréer l'effet voulu, en considérant l'identité du public cible ainsi que les aspects culturels et l'objectif de la communication. Cet article montre que ces deux lectures, respectivement raisonnée et émotive, s'avèrent essentielles pour l'opération à la fois rationnelle et créative qu'est l'adaptation publicitaire.

\section{ABSTRACT}

To reach and influence an audience, the advertising translator must master the art and science of advertising copywriting, especially since the advertisement's target audience and communication context might differ significantly from those of the source advertisement. As the cultural agent of an advertiser, the translator determines how every textual and paratextual component of the advertisement will undermine the communication process and make the required adjustments. To achieve this, the translator must do a double reading of the source ad in order to understand each communicatory issue and subsequently guide his process. First, the analytic reading corresponds to a rational decoding of the message, influenced by the translator's knowledge and skills in advertising copywriting as well as his work environment, the available resources and the communication context. Secondly, the empathetic reading allows the translator to understand how the source text works from the emotional viewpoint and ultimately to recreate the desired effect, taking into account the identity of the target audience as well as the cultural aspects and the objective of the communication. This paper argues that these two readings, respectively rational and emotional, are essential to the equally rational and creative activity that is the adaptation of advertisements.

\section{MOTS-CLÉS/KEYWORDS}

traduction publicitaire, lecture, adaptation, empathie, marketing advertising translation, reading, adaptation, empathy, marketing 


\section{Introduction : adaptation publicitaire et primauté du public cible}

Leo Burnett, fondateur d'une importante agence de publicité qui porte toujours son nom $^{1}$, a légué un héritage considérable dans l'imaginaire collectif occidental avec des créations qui ont rapidement intégré la culture populaire moderne: le Géant Vert, Tony le tigre, le petit bonhomme Pillsbury, sans oublier le célébrissime cowboy Marlboro, pour n'en citer que quelques-unes ${ }^{2}$. Fin connaisseur de la psychologie et de la communication humaines, Burnett a su orchestrer de grandes campagnes publicitaires en concentrant ses efforts non pas sur le produit ou sur l'annonceur lui-même, mais bien sur le récepteur du message. Le succès des campagnes publicitaires repose alors sur la capacité des concepteurs, rédacteurs et stratèges impliqués à se projeter dans la peau du récepteur afin d'en comprendre les goûts, les rêves, les opinions, les motivations et les éventuelles réactions à différents stimuli publicitaires. La clé est ici l'identification de la cible, qui doit se reconnaître en un clin d'œil dans le message et, d'une certaine façon, avoir l'impression que l'annonceur s'adresse particulièrement à elle. Ce phénomène psychologique est bien connu en théorie du marketing.

Source likeability, attractiveness, and similarity to the viewer all enhance message effectiveness by causing viewers to like and adopt the message delivered. [...] Early research shows that favorable similarity effects (that viewers prefer ads showing characters that are similar to them over ads containing characters that are different from them) are mediated by identification processes. (Brumbaugh 2009: 972)

C'est grâce à la capacité de se glisser dans les souliers des cibles, pour paraphraser Burnett, que les concepteurs parviennent à rejoindre et à toucher ces individus, pour ensuite modifier leur comportement, les pousser à passer à l'action ou à changer de point de vue sur une cause ou un produit donné.

Cette orientation vers le public cible entraîne bien entendu des changements importants dans le processus de réflexion qui précède toute activité créative et stratégique, et ce, pour tous les experts impliqués dans la communication publicitaire, qu'il s'agisse de rédacteurs, de directeurs artistiques, d'infographistes ou encore de traducteurs, dans le cas des campagnes internationales. Il est d'ailleurs particulièrement important pour les traducteurs de bien maîtriser les mécanismes de la communication publicitaire, car leur mandat est entre autres de décoder les différentes composantes d'une publicité en fonction d'un public de la culture cible, qui peut avoir bien peu en commun avec le public cible de la publicité source. Cela signifie que le traducteur publicitaire doit à la fois s'éloigner du contenu original et apporter une contribution résolument créative au processus de communication publicitaire multilingue ${ }^{3}$, et servir d'agent culturel entre un annonceur et une culture qui lui est étrangère. Ce rôle s'avère d'autant plus important de nos jours, alors que les agences de publicité voient, parfois malgré elles, un bon nombre de leurs mandats de création devenir des mandats d'adaptation, en raison de la mondialisation, de la progression du commerce international et des évidentes économies que cette pratique entraîne. Effectivement, même si elle implique un travail de traduction, de réécriture, d'infographie, de montage, de tournage, d'enregistrement et bien d'autres, l'adaptation sera normalement toujours moins coûteuse que la conception d'une campagne originale dans chaque culture visée (qui requiert normalement plus de temps ainsi que plus de ressources humaines et matérielles). Bref, le traducteur impliqué dans la stratégie de 
communication publicitaire bénéficie généralement d'une liberté assez importante pour que, malgré un ensemble complexe de contraintes sémantiques, culturelles et techniques, la publicité traduite connaisse des résultats semblables dans les cultures source et cible. Ce n'est pas un hasard si la traduction publicitaire est un des rares domaines où l'adaptation fait consensus, tant chez les praticiens que chez les traductologues: la nature persuasive et séductrice de ce type de communication explique ce biais très marqué pour l'approche ethnocentrique.

Les traducteurs se sont rendu compte de l'inadéquation du critère de fidélité pour ce genre de textes, dont le contenu et le but n'avaient rien de sacral pour exiger un respect scrupuleux de la forme ni même du fond. De ce constat de décalage entre la visée du message et le type d'équivalence recherchée naît la consigne d'adaptation pour les textes publicitaires. (Guidère 2009: 420)

Ce processus d'adaptation n'est pas qu'intuitif. Il s'appuie sur une lecture informée du traducteur puis sur une analyse influencée par ses connaissances en matière de marketing et de communication publicitaire ainsi que par son propre bagage culturel (qui est normalement celui du public visé).

Toutes les modifications ou interventions réalisées par le traducteur sont justifiées afin d'obtenir le même effet dans la culture cible. Ces modifications, loin d'être des prises de liberté aléatoires, sont des options guidées par un souci de respect de l'univers de référence de la culture visée et de la fonction de traduction. (Cómitre Narváez 2004: 88)

Cette importance de l'étape de la lecture n'est plus à démontrer en traductologie, la traduction elle-même étant pratiquement une combinaison cyclique des actes de lecture et d'écriture:

la lecture rythme le déroulement du processus, tant dans l'actualisation du texte, la compréhension, que dans la recherche de sources textuelles qui permettent de situer le texte à traduire, ou qu'au cours même de l'écriture où la lecture joue comme moyen d'évaluation et de distanciation critique du traducteur par rapport à son propre texte. (Plassard 2007: 22)

Cela dit, ces lectures sont particulièrement importantes en adaptation publicitaire, car le traducteur, en tant que premier récepteur d'un message étranger destiné à sa culture, joue d'une certaine façon le rôle de cobaye. C'est à ce titre qu'il doit repérer les arguments, les réflexions, les références ainsi que les éléments textuels et visuels qui nuiront à la compréhension rapide et à l'efficacité du message au sein de la culture cible, ou même qui risquent de choquer et de ternir l'image de l'annonceur. La définition de l'adaptation en traduction, proposée par Bastin, comme "processus d'expression d'un sens visant à rétablir un équilibre communicationnel rompu par la traduction» (1993: 477), correspond d'ailleurs parfaitement à cette réalité. Ainsi, cette lecture analytique du traducteur sera la première étape d'une opération d'adaptation linguistique et culturelle qui va ressembler en plusieurs points au processus créatif mené par les concepteurs de la publicité source. Elle sera toutefois suivie par une seconde lecture, cette fois d'ordre plus altruiste. En effet, si le public cible d'une opération de marketing fait d'une publicité une lecture égoïste (où son unique préoccupation est de savoir ce qu'il peut retirer du message, comment il peut en bénéficier), le traducteur, lui, doit plutôt en faire une empathique. Nous verrons plus loin comment ces étapes essentielles de lectures analytique et empathique façonnent le 
discours publicitaire dans la culture cible et comment l'apport créatif du traducteur s'articule autour d'elles, toujours dans l'optique d'une communication destinée à un public bien précis. Combinées, les lectures analytique et empathique permettront au traducteur de voir la publicité à adapter sous l'œil du public ciblé, et d'en anticiper la réception et la compréhension.

\section{Lecture analytique, identité et environnement de travail du traducteur publicitaire}

La persuasion publicitaire est un phénomène complexe dont la compréhension du fonctionnement implique des principes de psychologie, d'anthropologie, de sociologie, de commerce, de marketing et de consommation ${ }^{4}$. Le traducteur publicitaire doit considérer ces principes pendant la première lecture du texte à traduire afin, notamment, de percevoir les mécanismes du message (affectifs, incitatifs, mnémoniques, etc.) puis de les recréer de manière à conserver leur efficacité auprès du public de la culture cible, tant en ce qui concerne le fond, la forme et le contexte communicationnel. Cette lecture analytique a d'ailleurs beaucoup en commun avec l'élaboration du sens du texte de la théorie interprétative où, selon Seleskovitch et Lederer, la traduction est une opération axée non pas sur la langue, mais plutôt sur le sens, qui s'opère en deux étapes, à savoir la compréhension du message à traduire, puis sa reformulation par le traducteur:

The interpretive theory takes into account the general psychological processes of the understanding and production of discourse and the function of both source and target texts, and underscores the role played by translators in carrying sense across language barriers. (Lederer 2007: 21)

Il est facile d'établir un lien entre l'accent sur le lecteur cible et sur l'acceptabilité de la traduction dans la culture visée mis en évidence par la théorie interprétative et la fonction de la communication promotionnelle. Comme l'écrit Lederer, ceux qui requièrent la traduction de textes pragmatiques «are not interested in how a foreign language expresses things but in what it expresses, in its informational content» (2007: 26). Par ailleurs, cette idée de la réexpression du sens du message source demeure centrale en traductologie et ses implications pour le travail du traducteur ont été analysées sous bien des angles pertinents pour l'adaptation publicitaire, dont celui de la sociologie.

Le sens d'un énoncé repose sur deux types de composantes: le linguistique et l'extralinguistique. À l'intérieur du linguistique, on peut distinguer entre le ponctuel, qui concerne le signe et le structural, et le discursif, qui concerne la construction des énoncés, mais il faut reconnaître que la distinction n'est pas toujours aisée et qu'elle est même parfois non pertinente, car les deux éléments sont souvent liés. À l'intérieur de l'extralinguistique, il faut distinguer entre ce que l'on pourrait appeler des normes civilisationnelles et des connaissances. Pour qu'il y ait construction du sens, il importe que tous ces éléments soient réunis et que, de plus, il y ait un lecteur pour les percevoir et les assembler. (Ballard 1998: 27-28)

L’examen de chaque élément textuel et paratextuel du message mené pendant la lecture analytique requiert que le traducteur publicitaire connaisse les mécanismes et rouages de la communication publicitaire, tant des points de vue créatif et straté- 
gique que technique et pratique. De plus, différents facteurs interdépendants et très variables d'un mandat à l'autre influent sur le processus de lecture analytique du contenu de la publicité et l'alimentent. D'abord, les deux premiers facteurs qui influent sont directement liés au traducteur lui-même. Il s'agit des connaissances et aptitudes $d u$ traducteur en rédaction publicitaire ainsi que de l'environnement de travail $d u$ traducteur et des ressources à sa portée (personnes-ressources, outils et documents de référence, notes de travail des concepteurs du message original, etc.). Enfin, le troisième facteur est le contexte communicationnel et l'identité de l'annonceur, qui concerne entre autres son image de marque, son positionnement dans la culture cible, les contraintes techniques du média retenu et la visée de la traduction.

Les deux premiers facteurs dépendent du rôle que l'on donne au traducteur dans le processus de communication interculturelle et multilingue, et de son mandat précis pour chaque activité de marketing. Il importe donc de s'intéresser aux réalités de l'industrie de l'adaptation publicitaire pour comprendre comment diverses préoccupations socioéconomiques affectent les traducteurs, leur production et plus particulièrement leur lecture des messages à transmettre dans leur propre culture. D'abord, l'idée de communiquer un seul et unique message est alléchante pour bien des entreprises multinationales. En plus de permettre d'importantes économies de temps et d'argent, cette pratique assure un meilleur contrôle de l'identité de la marque et facilite grandement le façonnement de la perception d'une identité et d'une image cohérentes chez les différents publics cibles. Cependant, il a rapidement été démontré que, même dans ce «village planétaire», les idées ne voyagent pas aussi facilement, et les exemples de campagnes et de slogans ayant connu des échecs retentissants ou ayant nécessité des ajustements majeurs ne sont pas rares. Citons en exemple la White Power, version modifiée d'une voiture toute blanche de marque allemande ${ }^{5}$ ou le slogan du jeu vidéo Zelda Ocarina of time "Willst thou get the girl? Or play like one?», mal reçu dans certaines cultures pour sa connotation sexiste (de Pedro Ricoy 2007: 270-271). La dynamique de la communication publicitaire dans le contexte de la mondialisation évolue donc rapidement: «the earlier trend towards a homogeneous, international marketing approach in all markets has lost much of its appeal as companies have realised that imposing a single strategy may have an adverse effect» (Smith 2008: 47). En d'autres termes, un rédacteur new-yorkais, parisien, londonien ou californien ne peut prétendre savoir comment son message sera perçu dans des cultures dont il a une connaissance rudimentaire, sinon stéréotypée. La valeur de la contribution des traducteurs devient évidente: alors que nous assistons à ce que certains décrivent comme une «américanisation " planétaire des différentes cultures, les besoins de communication multilingue n'ont jamais été aussi importants. Simon note d'ailleurs que «the globalization of culture means that we all live in "translated" worlds» (1996: 127). Les traducteurs voient donc leur profession finalement valorisée au sein d'une industrie qui s'en est très souvent méfiée (avec la crainte, parfois fondée, que les textes traduits puissent être plus ternes et moins vivants, ou encore que les traducteurs soient dépourvus de la plume vivante, séductrice et astucieuse des rédacteurs publicitaires chevronnés). Toutefois, les traducteurs qui saisissent bien la culture publicitaire, qui maîtrisent bien les techniques de rédaction en marketing et qui savent faire preuve de créativité parviennent souvent à gagner rapidement la confiance de leurs nouveaux collègues. Comme le note Guidère, 
[1]e traducteur accède progressivement à de nouvelles fonctions et assume des responsabilités qui débordent largement le cadre de la traduction à proprement parler. Ainsi, par exemple, il participe à l'adaptation de campagnes publicitaires, intervient au sein des entreprises pour préparer les communications en langue étrangère, prend en charge la localisation des sites web en plusieurs langues, conseille les institutions et les organisations internationales sur la meilleure communication à adopter à l'égard de tel ou tel public cible, etc. (Guidère 2011 : 337)

Il apparaît par ailleurs évident que l'intégration et l'implication active des traducteurs au sein même des unités créatives multidisciplinaires de l'agence soient bénéfiques non seulement pour le développement de leur expertise en tant que professionnels de la communication publicitaire et promotionnelle multilingue, mais également pour la production complète et la diffusion du message traduit, bien audelà des étapes d'ordre textuel. Dans un article qui porte justement sur cette intégration des traducteurs au sein des unités créatives et stratégiques des agences de communication marketing, nous notons que:

[c]ette dynamique d'échange et de proximité permet au traducteur de bénéficier d'une plus grande marge de manœuvre pour trouver des solutions créatives et efficaces aux problèmes traductionnels de tout ordre, et de produire un document d'arrivée dont la cohésion avec les objectifs des clients et partenaires est totale. (Vandal-Sirois 2011:2)

Ainsi, le traducteur en agence profite d'une variété de ressources humaines et matérielles qui lui permettent de reproduire à peu de chose près le processus de création du message original, d'enrichir sa réflexion de conseils d'experts qui orienteront le travail de traduction et d'effectuer un suivi plus efficace de sa contribution lors des étapes qui suivent la traduction. Pensons par exemple à la manipulation et à la mise en page du texte traduit par des infographistes, ou aux stratèges en placement média qui décideront quels sont les meilleurs moyens de toucher le public pour lequel la publicité a été adaptée.

L’ensemble des publicités traduites et diffusées précédemment, une analyse des principaux concurrents présents dans le marché ciblé, des études de marché, et même des rapports de groupes de discussion (focus groups) sont des exemples d'outils de travail essentiels qui orientent et exercent une grande influence sur l'opération d'adaptation, et sur lesquels le traducteur pigiste, non intégré à l'agence, peut rarement mettre la main. (Vandal-Sirois 2011: 7)

Il ne s'agit ici que de quelques exemples de ressources qui orienteront la lecture analytique, liée à la compréhension du message par le traducteur puis à l'opération d'adaptation dans son ensemble. Il est intéressant de noter que le traducteur publicitaire peut parfois se retrouver dans des situations difficilement imaginables dans d'autres domaines de la traduction spécialisée. C'est d'ailleurs le cas avec les groupes de discussion que nous venons de mentionner, où la traduction est testée auprès de consommateurs appartenant à la culture cible. Le traducteur peut ainsi assister à la réception de son adaptation, alors que les récepteurs verbalisent spontanément leur compréhension du message traduit, ce qui lui permet de valider ses décisions puis, à long terme, d'en apprendre davantage sur le public qu'il vise. Inutile de préciser que ce genre d'exercice, bien que très rare dans les autres domaines de traduction, pour des raisons financières évidentes, est extrêmement enrichissant pour le traducteur qui souhaite approfondir ses aptitudes de lecteur. D’ailleurs, cette situation mériterait 
qu'on s'y intéresse davantage, car elle permet une évaluation qualitative et quantitative de la réception des traductions dans une culture donnée, dans une perspective relativement unique en traductologie. Toujours est-il que la lecture analytique sera grandement influencée par l'expérience du traducteur au fait de différentes notions de marketing, ainsi que par la place qu'il occupe au sein du processus communicationnel entre un annonceur et son public cible.

Simultanément, la lecture analytique implique une réflexion concernant le contexte communicationnel et l'identité de l'annonceur. Encore une fois, le traducteur doit nécessairement considérer ces facteurs extratextuels pendant la phase de compréhension et de décodage du message publicitaire, mais aussi au moment où il en rédige la version adaptée. Le ton, le style, le registre de la langue et les cas de synonymie, par exemple, seront tous régis par l'identité de l'annonceur et son positionnement dans le marché cible. Le produit ou la cause sont-ils autant connus dans la culture cible que dans la culture source? Le public cible est-il habitué à une marque concurrente qui n'existe pas dans le marché source? Les réponses à ces questions peuvent entraîner un travail d'ajustement, ou même de réécriture, qui sera souvent effectué par le traducteur, en tant qu'expert de la culture cible. Dans le même ordre d'idées, le traducteur devra utiliser un style qui s'harmonise à l'image de marque que souhaite projeter l'annonceur. C'est pourquoi certains produits seront respectivement «robustes», «solides» ou encore «durs comme le roc». L'utilisation du pronom «you» dans la langue publicitaire anglo-saxonne illustre bien l'importance pour le traducteur d'effectuer une lecture analytique approfondie au lieu d'opter pour le choix évident du vouvoiement, qui n'est pas invariablement la meilleure solution, notamment lorsqu'on s'adresse à un public plus jeune.

De plus, pour produire des textes efficaces et cohérents avec le client et sa marque, les traducteurs devront parfois utiliser les mêmes outils et techniques que les rédacteurs, et surtout orienter leurs décisions traductionnelles. De tous ces éléments propres à la stratégie marketing qui inspirent et balisent le processus créatif, l'axe de communication compte certainement parmi les plus utiles pour les traducteurs. Cet axe est le fil conducteur des activités de communication, l'idée centrale qui doit revenir de manière cohérente dans tous les messages, sous toutes les formes. L'axe représente de manière cohérente et constante l'annonceur, son identité et ses promesses. En fait, la notion bien connue en marketing d'Unique Selling Proposition (USP) s'en rapproche, dans la mesure où il s'agit de déterminer les particularités de l'entreprise, qui expliquent en quoi elle se distingue de la concurrence. Selon Dagenais, l'axe «doit orienter tous les messages. Ceux-ci peuvent conjuguer l'axe de diverses façons, mais ils ne peuvent s'en éloigner» (1998: 308). Il est ainsi impératif que chaque individu impliqué de près ou de loin dans les activités de communication d'une organisation en connaisse l'axe, afin de présenter un message constant et de respecter l'image que l'annonceur a développée au fil du temps. Le contexte communicationnel comprend également les différentes contraintes formelles et techniques du média de communication retenu, avec lesquelles le traducteur doit composer. Il peut s'agir de particularités visuelles et typographiques qui obligent le traducteur à trouver une adaptation convenable pour un jeu de mots lié à une image, ou à travailler en collaboration avec un infographiste, ou encore de limites spatiales ou temporelles qui lui sont imposées. Certains annonceurs ont le mauvais réflexe de vouloir maximiser l'espace média acheté en plaçant le plus de données possible (qu’elles soient 
sonores ou visuelles), ce qui peut compliquer la tâche du traducteur, selon notamment les combinaisons de langues de travail.

Le contexte de réception aura également une grande importance: le lecteur peutil lire le message adapté à son propre rythme ou ne dispose-t-il que de quelques secondes, comme dans le cas des panneaux sur le bord de l'autoroute? Le message aura-t-il toute l'attention du récepteur, ou sera-t-il diffusé par le biais d'un média d'accompagnement comme la radio? De plus, dans le cas des messages télévisés, radiophoniques et pour le Web, il faut ajouter à ces considérations les contraintes habituelles de la traduction audiovisuelle. Bref, les résultats de la lecture analytique représentent les données concrètes et observables qui serviront tant de guide que de muse pour le traducteur publicitaire qui, pour compléter la réflexion préalable à la traduction publicitaire, devra ensuite opérer une lecture empathique, plutôt d'ordre qualitatif, émotionnel et intuitif.

\section{Lecture empathique, public cible et considérations fonctionnalistes en traduction publicitaire}

À l'instar de la lecture analytique, la lecture empathique est une étape du processus traductionnel en publicité, qui permet d'optimiser l'efficacité du message adapté auprès du nouveau public cible. Il s'agit ici pour le traducteur de se mettre à la place de sa cible et de s'identifier à elle en considérant ses intérêts, ses habitudes, ses problèmes, ses rêves, son environnement, etc., pour anticiper la perception du message et en accroître par le fait même l'efficacité. La lecture empathique permet ainsi au traducteur de déterminer si ses cibles auront la réaction souhaitée par l'annonceur, qu'il s'agisse d'une réaction identique à celle suscitée par le message original ou non, et guidera les décisions traductionnelles en conséquence. Dans un article sur l'« empathie rationnelle» chez les traducteurs, Collombat écrit que cette empathie devrait les conduire

à savoir quand adopter tel code linguistique et pourquoi, sans préjugé et sans se laisser guider par d'autres principes que par le souci de s'acquitter de son mandat en toute objectivité et avec professionnalisme. (Collombat 2010: 67)

De plus, elle avance que:

la compétence technique est indépendante des compétences empathiques - soit l'ensemble des compétences interrelationnelles - mais complémentaire de celle-ci, formant avec elle un ensemble indissociable pour la réalisation optimale du mandat. (Collombat 2010: 60)

La lecture empathique se divise elle aussi en trois facteurs dont les frontières se chevauchent: identité et caractéristiques du public cible, aspects culturels de la communication et objectif de la communication. C'est en considérant simultanément ces trois facteurs que le traducteur comprendra comment le message opère chez les cibles et qu'il pourra ensuite reproduire l'effet voulu, en connaissant d'emblée l'ampleur des modifications à apporter au message. En d'autres mots, cette lecture aide le traducteur à justifier logiquement ses décisions à ses clients et collègues, alors qu'il est confronté à des éléments difficilement quantifiables, souvent d'ordres émotionnel et instinctif. À ce propos, le traducteur devra se méfier de ses propres réactions face à un texte qui 
est justement conçu pour émouvoir, surprendre, faire rire ou même choquer les receveurs, et surtout s'il est confronté à un message politique, sociétal ou promouvant des produits controversés.

De plus, cette distanciation peut s'avérer particulièrement difficile, à partir du moment où chaque composante du produit cherche à capter puis à retenir l'attention du récepteur et à favoriser son identification à la promesse annoncée. Les choix du traducteur seront alors d'autant plus importants, car ils guideront la compréhension des lecteurs ciblés de façon définitive. Il doit ainsi s’assurer que les émotions créées soient conformes à l'objectif de la communication et orientent bien la cible de la façon voulue par l'annonceur. Cela illustre l'efficacité de l'adaptation comme stratégie de marketing multilingue, et la force d'une communication conçue sur mesure pour la personne à qui l'on s'adresse: "Effective adaptations can both enlarge and amplify the "truth" of whatever is being adapted...» (Welsh 2011: 17).

Dans le même ordre d'idées, les notions d'identité et de caractéristiques du public cible sont centrales à l'activité de lecture empathique, car c'est à l'égard de ce public précis que le traducteur devra justement faire preuve d'empathie, afin de s'assurer que le message soit capté, compris et assimilé. Pour y parvenir, il devra se glisser dans les souliers de ces consommateurs cibles, pour reprendre l'expression de Leo Burnett. Cette faculté d'empathie est particulièrement importante aujourd'hui, alors que les auditoires sont de plus en plus fragmentés et que le nombre de médias spécialisés croît rapidement. Les annonceurs délaissent les médias de masse pour une communication beaucoup plus ciblée et, dans certains cas, personnalisée. Comme le résume Dan Rosensweig, directeur de l'exploitation chez Yahoo! de 2002 à 2007: « The world is moving from mass media to my media» (Roberts 2006:38). Alors qu'on se contentait auparavant de cibler un public large, comme les baby-boomers, la plupart des initiatives de marketing d'aujourd'hui visent plutôt les «mères monoparentales de 25 à 35 ans, de classe moyenne, avec un baccalauréat» ou les «travailleurs des technologies de l'information qui habitent au centre-ville». Cette tendance marquée vers le ciblage de publics de plus en plus pointus correspond par ailleurs à la place de plus en plus imposante que prennent Internet et les réseaux sociaux dans les stratégies de marketing, car ils permettent de toucher des groupes de cibles restreintes en nombre, mais très précises.

Ainsi, le traducteur doit savoir précisément à qui il s'adresse, et devra s'interroger sur l'ampleur des différences entre les publics des publicités source et cible. Même lorsque les consommateurs visés sont du même sexe et du même groupe d'âge, par exemple, les différences linguistiques et culturelles peuvent justifier à elles seules des adaptations majeures. Le traducteur publicitaire en agence possède une longueur d'avance, car il peut souvent profiter des descriptions de publics visés par les créateurs du message original, un point de départ idéal pour sa propre démarche, et même de données démographiques pertinentes sur les consommateurs de la culture cible. Toutefois, pour être certain de prendre la bonne décision lorsqu'il est confronté à certaines difficultés traductionnelles, il a la responsabilité de pousser sa réflexion bien au-delà de la simple consultation de tels documents. Selon Dagenais (1998: 156-158), pour bien identifier le public ciblé, tout communicateur doit se poser quatre questions : qui sont-ils? (âge, revenus, scolarité, lieu de résidence, etc.), que savent-ils, que pensent-ils? (attitudes, méfiances, motivations, influences, etc.) et que font-ils? 
(habitudes de consommation et comportement). La connaissance des différentes facettes de son public permettra au traducteur de toucher ses cibles et de leur donner l'impression que le message a été conçu sur mesure pour elles. C'est d'ailleurs ici que le travail effectué lors de la lecture analytique, particulièrement en ce qui concerne le contexte communicationnel et l'identité de l'annonceur, commence à aiguiller la lecture empathique. Après tout, les conditions d'émission d'un message ont généralement un impact considérable sur sa réception. L'appartenance du traducteur à la culture s'avère impérative, car s'il n'appartient pas exactement au public visé, il coexiste avec celui-ci et le côtoie dans certains cas. La lecture empathique n'en est que facilitée.

Ensuite, et de manière complémentaire à la réflexion sur l'identité du public, le traducteur doit considérer les enjeux culturels dans sa lecture empathique d'une publicité à adapter. La culture est effectivement un concept clé, qui oriente les choix de conception publicitaire puis d'adaptation du message créé, occupe une place prépondérante dans la formation des professionnels de la communication et dans la recherche universitaire en marketing:

The best international marketers will not only appreciate the cultural differences pertinent to their businesses, but they will also understand the origins of these differences. Possession of the latter, deeper knowledge will help marketers notice cultural differences in new markets and foresee changes in current markets of operation. (Cateora, Gilly, et al. 2011: 103)

À l'instar des concepteurs-rédacteurs, les traducteurs doivent donc savoir tirer profit du lien très fort entre une culture et sa langue pour produire des adaptations efficaces. Le traducteur a donc la tâche de déterminer le compromis parfait entre le propos de l'annonceur et l'identité socioculturelle du public cible, alors que le moindre déséquilibre rend la communication inopérante: «si les prémisses culturelles dont part l'argumentation publicitaire ne jouissent pas de l'adhésion du destinataire, [...] le message perd en fonctionnalité» (Ventura 2009: 462). Cette fonctionnalité, l'unique raison d'être de la publicité originale et de son adaptation, constitue d'ailleurs le troisième facteur de la lecture empathique.

Pendant la lecture et la rédaction de son adaptation, le traducteur ne doit jamais perdre de vue l'objectif de la communication. Une publicité est une création calculée et intéressée, conçue spécifiquement dans le but de modifier le comportement du récepteur, ou d'en influencer l'opinion. Si l'idée d'adapter un texte d'après le public ciblé est davantage acceptée en traduction publicitaire qu'en traduction littéraire, par exemple, c'est justement parce qu'il n'y a plus d'auteur à trahir, mais seulement un mandat bien précis à remplir. Cet objectif de communication se situe au cœur de la notion de Skopos de Vermeer, qui stipule que le traducteur doit interpréter et rédiger ses textes «in a way that enables [his] text/translation to function in the situation in which it is used and with the people who want to use it and precisely in the way they want it to function» (Nord 1997: 29).

Lorsqu'une entreprise fait adapter une publicité pour un marché cible, elle ne souhaite pas faire connaître le contenu du message original dans une autre nation. Elle veut plutôt faire connaître puis vendre ses produits aux consommateurs de la culture cible, tout simplement. Dans cette optique, le lien entre les objectifs communicationnels d'une campagne multilingue et la théorie fonctionnaliste en traducto- 
logie est évident: la visée du texte, l'identité et la motivation de l'employeur, le contexte communicationnel et les caractéristiques du public cible sont tous des exemples d'éléments considérés à la fois par le traducteur qui adopte l'approche fonctionnaliste et par le concepteur publicitaire. Ces éléments à considérer sont compris dans ce que Nord appelle le «brief» de la traduction, un terme que la théorie fonctionnaliste et le monde de la conception publicitaire ont d'ailleurs en commun (Nord 1997: 59-62). Toutefois, cette théorie résolument cibliste ne recommande pas au traducteur d'opter systématiquement pour l'adaptation, mais bien pour le type de traduction qui convient le mieux à l'objectif de cette traduction, quel qu'il soit:

What the Skopos states is that one must translate, consciously and consistently, in accordance with some principle respecting the target text. The theory does not state what the principle is: this must be decided separately in each specific case. (Vermeer 2000: 228)

C'est alors au traducteur à déterminer le Skopos de son mandat, seul ou avec ses collègues créatifs, d'après les facteurs traductionnels et communicationnels pertinents.

La faculté d'empathie envers le lecteur ciblé est précieuse pour le traducteur publicitaire, qui doit choisir les bons mots, le bon style, voire les bons arguments afin que les récepteurs ressentent l'effet voulu par la stratégie communicationnelle. La lecture empathique permettra donc au traducteur d'unir de manière cohérente et rationnelle l'ensemble des facteurs considérés pendant la lecture analytique, et facilitera par conséquent l'exercice parfois périlleux mais toujours efficace de bondir «dans les souliers» des récepteurs du message.

\section{Synthèse et applications pratiques}

Ainsi, les lectures analytique et empathique sont deux étapes essentielles pour l'opération à la fois rationnelle et créative qu'est l'adaptation publicitaire. La première lecture, stratégique, permet de déterminer quel sera le message à adapter, quel est le contexte de communication (situation de l'annonceur et de la cible) et quels seront les obstacles à cette communication interculturelle. Elle est modelée par les connaissances et les compétences en publicité et en marketing du traducteur, ainsi que par son environnement de travail et les ressources matérielles et humaines dont il dispose. Ensuite, le traducteur effectue une seconde lecture du texte à traduire, teintée par les conclusions de la lecture analytique, en se mettant dans la peau de la cible afin d'en anticiper les éventuelles réactions face aux différentes orientations traductionnelles retenues. Au cours de cette lecture, le traducteur mise sur sa connaissance du public cible et sur son propre bagage culturel afin de mettre le doigt sur les meilleures solutions pour optimiser la réception du message, son impact, sa rétention et son efficacité, dans le simple but avoué que la cible change d'avis ou, plus fréquemment, qu'elle passe à l'action. Il est possible de schématiser ce processus de double lecture de la façon suivante: 


\section{FIGURE 1}

\section{Lectures analytique et empathique en traduction publicitaire}

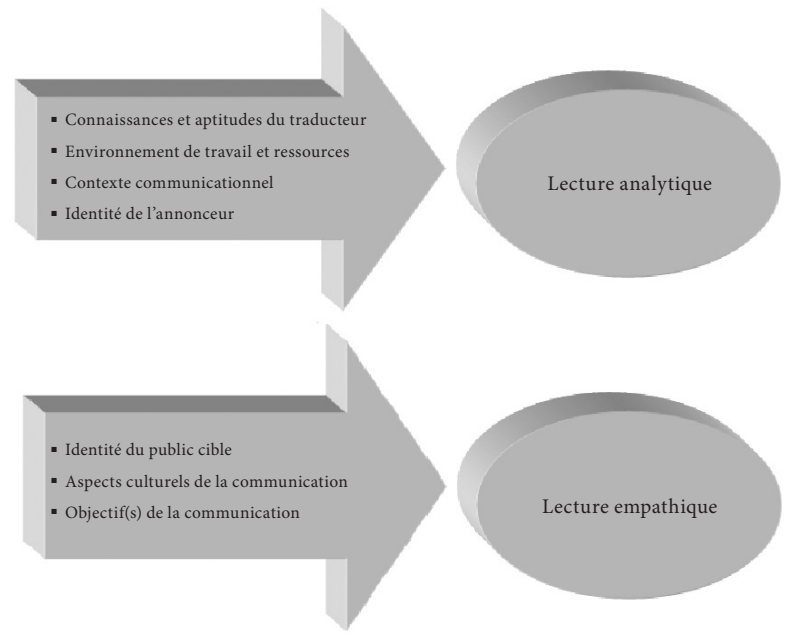

Les facteurs de lecture analytique et empathique n'affecteront pas tous l'opération de traduction de la même façon, car chaque communication publicitaire correspond à un amalgame de variables: quel est l'annonceur? Quel est le produit ou l'idée mise de l'avant? À quels besoins ce produit répond-il? Quels sont la personnalité de la marque et le public visé? Quel est le contexte de communication? En quoi diffère-til du contexte du message original? Quels sont les obstacles à l'atteinte des objectifs communicationnels? Etc. Le traducteur publicitaire doit alors départager les facteurs pertinents de ceux qui n'influenceront pas la réception du message, sans oublier que son propre public évolue dans une réalité culturelle, sociale et économique différente de celle des concepteurs du message initial. Prenons par exemple Bombardier Produits Récréatifs, dont la signature a longtemps été "Chez nous, c'est Ski-Doo». La lecture analytique de cette ligne permettra de déterminer que la compagnie québécoise a décidé de séduire les consommateurs francophones en misant sur son origine (et celle de l'inventeur de la version moderne de la motoneige). L'appel au sentiment d'appartenance à une entreprise québécoise bien établie mondialement, accentué par l'expression rassembleuse et familière "chez nous", s'est avéré particulièrement efficace, et touche une corde sensible chez le public visé, d'autant plus que ce sport motorisé se pratique souvent entre amis, ou au sein de clubs communautaires. Stratégiquement, Ski-Doo se positionne ainsi comme la marque de choix des motoneigistes québécois, comparativement aux produits de la compétition européenne ou américaine. Ces éléments liés au contenu du message, à la stratégie de marketing retenue et au contexte de communication sont alors complétés par une analyse de la forme du message. Dans le cas de cette signature, on note l'utilisation d'une formule idiomatique d'une grande oralité et une assonance évidente entre «nous» et «Ski-Doo». Ensuite, pendant la lecture empathique subséquente, le traducteur anglophone à qui on demande d'adapter cette ligne pour le marché américain et canadien-anglais notera d'abord que le lien affectif (géographique et historique) entre la marque et le public de la culture source n'existe plus pour le public anglophone. En imaginant la réception des consommateurs pour qui il écrit, le traducteur 
peut aisément conclure qu'une adaptation proche de l'original n'entraînera pas du tout la même réaction. Il faudra considérer cette différence majeure, car elle est cruciale pour cette communication. Du point de vue identitaire, outre la barrière culturelle et linguistique, les deux publics partagent par contre plusieurs points en commun (revenus, éducation, habitudes de consommation des produits offerts, etc.). Ainsi, il n'est pas étonnant que l'adaptation retenue, There's nothing like it, adopte un ton et un registre de langue semblables. On a également conservé un aspect comparatif qui positionne Ski-Doo par rapport à ses concurrents, tout en évoquant davantage l'aspect unique de la marque de façon plus générale.

Un autre cas intéressant est celui des bonbons Maynards, dont les communications publicitaires se concluaient avec la signature Chews to smile. La lecture analytique (public visé, objectifs de la communication) permet ici de déterminer que l'entreprise met en évidence le plaisir simple et accessible de manger des confiseries. Tout dans le fond et la forme du message correspond à cet axe, des couleurs vives, à la typographie gonflée et dansante, sans oublier le jeu de mots (choose/chews). Pendant la lecture empathique, le traducteur doit considérer la fonction de la communication (créer une association entre la consommation du produit et l'idée d'un plaisir accessible et immédiat) et s'assurer, pour que la stratégie identifiée soit respectée et demeure opérante, que le public visé ressente le même effet. Ainsi, l'adaptation française, "C'est bonbon de sourire», présente également un aspect enfantin et ludique très présent, tout en rappelant clairement la promesse du produit, peut-être même de manière encore plus éloquente que la signature originale.

Si la traduction est à la fois une science et un art, ces deux lectures représentent chacun des volets de la pratique de l'adaptation publicitaire. Il n'est pas anodin que la conception et la rédaction publicitaires soient d'ailleurs régies par cette même dualité raison/émotion, rationnel/affectif. De plus, si la notion de lecture en traductologie s'applique bien à l'adaptation publicitaire, certaines théories de la communication sont quant à elles «compatibles» avec la réflexion traductologique, et il s'avère possible d'appliquer des modèles de création publicitaire à l'activité de traduction. Alors que les milieux professionnels de la traduction et de la création publicitaire se rapprochent de plus en plus en raison des réalités socioéconomiques du commerce international, il serait pertinent d'effectuer ce même rapprochement entre la traductologie et les nombreuses théories de la communication multiculturelle. Hatim, par exemple, a étudié le processus d'importation d'un texte au-delà des frontières de sa langue et de sa culture source (Hatim 1997) ainsi que l'importance des stratégies de communication contextuelles derrière le fond et la forme du texte (Hatim et Mason 1997). Les contributions des experts du marketing peuvent également inspirer les traductologues intéressés par l'adaptation et les professeurs qui donnent, entre autres, des cours de traduction publicitaire, commerciale ou même littéraire. Pensons notamment à Godin (2003) qui rappelle aux professionnels de la communication l'importance de concevoir des textes remarquables et qui suggère des moyens pour y arriver, ou encore à Sullivan (2008), qui s'attarde à la voix de la marque qu'il faut identifier et utiliser, et à la complémentarité parfois subtile entre l'image et le texte promotionnels, deux éléments que le traducteur publicitaire doit comprendre et maîtriser.

Par ailleurs, la projection du traducteur dans les souliers de la cible pour qui il écrit et plus particulièrement les concepts de lectures analytique et empathique décrits 
dans le présent article ne sont pas sans rappeler The Translator's Turn de Robinson, où ce ne sont plus les textes sources et cibles qui se situent au centre de l'activité de traduction, mais bien le traducteur lui-même. Il est vrai que la traduction est influencée par le bagage de celui qui l'opère (ses émotions, ses impressions, son éducation, sa culture, etc.), d'autant plus que Robinson parle de traduction en termes de réaction, d'intuitions et de transfert de feeling (Robinson 1997: 18), encore une fois des principes inhérents à la psychologie du marketing. Cette réorientation de l'axe traductif confère au traducteur l'autonomie et le pouvoir de prendre des initiatives et de donner au texte cible une couleur personnelle, ce qui n'est pas incompatible avec son expérience d'identification à la cible visée par la publicité. Somme toute, les facteurs paratextuels qui contraignent, renseignent, inspirent ou tout simplement influencent significativement le traducteur publicitaire sont abondants. Voilà pourquoi opérer une double lecture juste et efficace s'avère indispensable pour le traducteur dont l'objectif est de rédiger un message publicitaire longuement réfléchi, calculé et travaillé, mais qui sera pourtant perçu, compris, ressenti et assimilé en un clin d'œil par sa cible.

\section{NOTES}

1. Leo Burnett Worldwide. Consulté le 15 février 2014, <http:// www.leoburnett.com>.

2. Pour en savoir plus sur Leo Burnett et ses campagnes publicitaires, voir l'article «Leo Burnett, the Marlboro Man of ad agencies» de Phil Rosenthal, publié dans le Chicago Tribune, 4 août 2010. Consulté en ligne le 19 février 2015, <http://articles.chicagotribune.com/2010-08-04/business/ctbiz-0804-phil-burnett-20100804_1_leo-burnett-worldwide-jolly-green-giant-marlboro-man>.

3. Voir Guidère (2008).

4. Voir Adam et Bonhomme (2005) ou Ferrell et Hartline (2011).

5. ArCAND, Denis (30 avril 2009): Dérapage de traduction: la Audi WHITE POWER change de nom. La Presse. Consulté le 30 mai 2014, <http://auto.lapresse.ca/actualites/201109/12/01-4441212derapage-de-traduction-la-audi-white-power-change-de-nom.php>.

\section{RÉFÉRENCES}

Adam, Jean-Michel et Bonhomme, Marc (2005): L'argumentation publicitaire. Rhétorique de l'éloge et de la persuasion. Paris: Éditions Armand Colin.

BALlard, Michel (1998): Les «mauvaises lectures»: étude du processus de compréhension. In: Jean Delisle et Hannelore Lee-Jahnke, dir. Enseignement de la traduction et traduction dans l'enseignement. Ottawa: Les Presses de l'Université d'Ottawa, 27-47.

Bastin, Georges L. (1993): La notion d'adaptation en traduction. Meta. 38(3):473-478.

Brumbaugh, Anne M. (2009): Why do I identify with thee? Let me count three ways: How ad context influences race-based character identification. Psychology \& Marketing. 26(11):970986.

Cateora, Philippe, Gilly, Mary C. et Graham, John L. (2011): International Marketing. New York: McGraw-Hill/Irwin.

Collombat, Isabelle (2010) : L'empathie rationnelle comme posture de traduction. TranscUlturAl. $1(3): 56-70$.

Cómitre Narváez, Isabel (2004): Stratégies de traduction de l'ambivalence dans le texte publicitaire. Anales de filología francesa. 12:81-93.

DAgENAIs, Bernard (1998): Le plan de communication. L'art de séduire ou de convaincre les autres. Québec: Les Presses de l'Université Laval.

DE PEDRo Ricoy, Raquel (2007): Internationalization vs. Localization: The Translation of Videogame Advertising. Meta. 52(2):260-275. 
Ferrell, O. C. et Hartuine, Michael D. (2011): Marketing Strategy. $5^{\mathrm{e}}$ éd. Mason: SouthWestern Cengage Learning.

Godin, Seth (2003): Purple Cow: Transform Your Business by Being Remarkable. New York: Portfolio.

Guidère, Mathieu (2008): La communication multilingue. Traduction commerciale et institutionnelle. Bruxelles: De Boeck.

Guidère, Mathieu (2009): De la traduction publicitaire à la communication multilingue. Meta. 54(3):417-430.

Guidère, Mathieu (2011): Les corpus publicitaires: nouvelles approches et méthodes pour le traducteur. Meta. 56(2):336-350.

Hatim, Basil (1997): Communication Across Cultures: Translation Theory and Contrastive Text Linguistics. Exeter: University of Exeter Press.

Hatim, Basil et Mason, Ian (1997): The Translator as Communicator. Londres: Routledge.

LEDERER, Marianne (2007): Can Theory Help Translator and Interpreter Trainers and Trainees? The Interpreter and Translator Trainer. 1(1):15-35.

NoRd, Christiane (1997): Translation as a Purposeful Activity: Functionalist Approaches Explained. Manchester: St. Jerome.

Plassard, Freddie (2007): Lire pour traduire. Paris: Presses Sorbonne Nouvelle.

Roberts, Kevin (2006): The Lovemarks Effect: Winning in the Consumer Revolution. New York: powerHouse Books.

Robinson, Douglas (1997): The Translator's Turn. Baltimore: Johns Hopkins University Press.

Simon, Sherry (1996): Gender in Translation: Cultural Identity and the Politics of Transmission. Londres: Routledge.

Smith, Veronica (2008): Visual Persuasion: Issues in the Translation of the Visual in Advertising. Meta. 53(1):44-61.

Sullivan, Luke (2008): Hey, Whipple, Squeeze This: The Classic Guide to Creating Great Ads. Hoboken: Wiley.

Vandal-Sirois, Hugo (2011): Publicités multilingues: l'apport du traducteur en agence de communication marketing. ILCEA (Revue de l'Institut des langues et cultures d'Europe et d'Amérique). 14:2-11.

Ventura, Daniela (2009): Présent et futur de la traduction publicitaire: entre eikôs et pathos. Meta. 54(3):450-465.

Vermeer, Hans J. (2000): Skopos and Commission in Translational Action. In: Lawrence Venuti, dir. The Translation Studies Reader. Londres: Routledge, 221-232.

Welsh, Jim (2011): Adaptation Past, Present and Future: An Interview with Jim Welsh. Journal of American Studies of Turkey. 33-34:5-19. 